Navigating the athlete role: Identity construction within New Zealand's elite sport environment

Submitted to Qualitative Research in Sport, Exercise and Health

\author{
${ }^{a}$ University of Waikato, New Zealand \\ * University of Waikato \\ Private Bag 3105 \\ Hamilton 3240 \\ New Zealand \\ Phone; $+647838-4466$ (8472) \\ E-mail: christina.ryan@waikato.ac.nz
}

Christina Ryan ${ }^{\mathrm{a}^{*}}$ 


\section{Navigating the athlete role: Identity construction within New Zealand's elite sport environment}

The purpose of this study was to explore how involvement within New Zealand's elite athlete development programme - athlete carding - has impacted the identity construction of this elite athlete population. Semistructured interviews were conducted with a purposeful sample of 17 carded athletes from eight different sports, followed by inductive thematic analysis of emerging key themes. In order to ensure that participants' voices were accounted for throughout the data representation process, athlete's individual stories were collated into collective accounts and represented using direct quotes. The potential for participants to become engulfed within the athlete role was evident throughout each of the carded athletes' stories. In many cases this role was consolidated through expectations on athletes' time and energies, as well as external scrutiny and performance pressures resulting from their involvement within the carding system. Whilst many athletes appeared to passively accept an increasing emphasis being placed upon their athlete role identity, some seasoned participants questioned why the carding system encouraged this solitary existence at the expense of a more multi-dimensional sense of self. Results suggest that the carded athlete system it is not currently meeting its potential to develop world class 'holistic' athletes. As such, it is important that High Performance Sport New Zealand re-examines its policy of performance-based funding and engages in a wide-reaching education programme which promotes the importance of athletes developing a well-balanced sense of self alongside their sporting potential.

Keywords: athlete identity; athlete development; sport ethic; performance culture 


\section{Introduction}

Athlete identity consists of the cognitive, affective, behavioural, and social aspects of identifying with the athlete role (Brewer et al. 1993). Like all role identities, an athlete identity is developed through the process of constructing and interpreting meanings within various social interactions. In this sense, athletes use socially constructed norms, values and beliefs associated with the sporting role to help understand the world around them (Miller 2009, Weiss 2001). Over the years there has been considerable research examining the formation and impact of athlete identity, revealing a diverse number of sport-related identities across a variety of differing populations (e.g. Brewer et al. 1993, Carless and Douglas 2012, 2013, Cosh et al. 2013, Donnelly and Young 1998, Lavallee and Robinson 2007, Sparkes 1998). Many of these investigations have raised concern about the high numbers of athletes who become subsumed by the athlete role (Coakley and Donnelly 1999, Litchke et al. 2012, Louder 2003, Miller and Kerr 2003, Wilding et al. 2012).

Whilst not inherently unhealthy, such a singular identity focus can become problematic if not explored in conjunction with other role identities. Specifically, individuals who become engulfed within the athlete role often limit their involvement in other contextual domains such as education, career development or social networks. This identity narrowing is linked to social and emotional difficulties, and negatively impacts athletes' decision-making and readiness for post-sport careers (Albion and Fogarty 2005, Murphy et al. 1996, Sturm et al. 2011). Likewise, an exclusive focus on athletic identity has been shown to increase an athlete's vulnerability to anxiety, depression and low self-esteem (Carless and Douglas 2009, Stephan and Brewer 2007). Such risks are especially heightened when one's athletic identity becomes disrupted following an injury or unexpected end to their sporting career (Hughes and Coakley 1991, Wainwright et al. 2005, Webb et al. 1998). 
The strength of the athlete role can in part be attributed to the long hours of training and intense level of specialisation thought necessary to become successful in the sporting domain (Maguire 2004, Price et al. 2010, Wilding et al. 2012). Frequent training, team rituals and other social and fitness-related requirements can leave athletes with limited free time and physical and psychological energy to explore alternative role identities (Lally and Kerr 2005, Murphy et al. 1996, Wilding et al. 2012). Additionally, the high regard given for sporting prowess within society, means that athletic talent is often favourably nurtured by those around the athletic performer (i.e. coaches, parents, peers, etc.) (Butt and Molnar 2009, Hughes and Coakley 1991). This encouragement is said to be especially strong due to the fact that the attention and importance given to sporting feats remains unmatched in most other talent domains (Archer 2010, Brewer et al. 1993). Consequently, the athlete role has become a socially accepted, and in some respects a socially sought after, role enactment (Miller et al. 2007).

In order to fulfil this role many athletes are, however, urged to place their lives on hold for the sake of their sport. Some high performance sports programmes have been found to actively discourage young athletes from participating in activities outside of the sport environment, whilst others purposefully avoid discussions on such topics in order to ensure athletes maintain a clear sport-only focus (Aquilina 2013, Cosh and Tully 2014, Henry 2013, Ryan, 2015, Stephan and Brewer 2007). Hughes and Coakley (1991) suggest this behaviour is part of the "sport ethic", or social norms that emphasise the need to sacrifice in the name of being an elite athlete. They propose that the sport ethic has become so prevalent within society that it is now the underlying value system of sport itself. Likewise, Carless and Douglas (2013) described such expectations as part of the strong performance narrative which exists within elite sport culture. This narrative champions the importance of performance outcomes (i.e. winning) and 
the belief that prioritisation of such outcomes is the only way in which one can actually achieve this success (Carless and Douglas 2012). Much like Hughes and Coakley, these authors also suggest "such is the dominance of this type of story it often becomes totalitarian to the extent that performance stories are expected of all athletes" (2009, p. 54).

Despite widespread acceptance of this narrowed identity, participation in programmes which encourage athletes to explore opportunities away from the sport environment (e.g. education or employment), have not resulted in subsequent decreases in athletic performance (Albion and Fogarty 2005, Aquilina 2013, Price et al. 2010). Rather, some studies have actually suggested that developing one's personal and sporting self simultaneously fosters excellence that would not be possible with just a singular identity focus (Aquilina 2013, Carless and Douglas 2012, 2013, Henry 2013, Lally 2007, Miller and Kerr 2003). Likewise, Price et al. (2010) found that time away from the rigours of competitive sport provided athletes with both mental and physical rejuvenation. These results therefore draw into question the current sport ethic and suggest that athletic success may in fact benefit from being pursued in conjunction with a well-rounded approach to personal development (Carless and Douglas 2013).

However, whilst most sport psychologists now agree that sport environments should be promoting holistic athlete development, the prevailing performance culture continues to underpin many athlete development programmes (Pummell et al. 2008). One such programme is High Performance Sport New Zealand's (HPSNZ) carded athlete programme. Consequently, the current research project sought to examine the experiences of selected New Zealand carded athletes and explore how this elite sport environment has impacted the identity construction of this specific population. In so doing, it is hoped that HPSNZ, and those individuals 
implementing similar sport development programmes around the world, will discover ways to best meet the holistic needs of these athlete populations.

\section{Background}

Established in 2002, HPSNZ's carded athlete system is a talent selection and development programme which aims to improve the lives of New Zealand's high performance athletes. Athletes become part of the carding system after being identified as talented by their national sporting organisations (NSOs). Once carded, athletes are provided with varying levels of access to coaching, career education, sport science and medical services to aid in both their future athletic performances and their personal development outside of their sport (Ryan 2015). Every year selected NSOs are allocated with funding from HPSNZ, specifically earmarked to developing these elite athletes. NSOs then decide how this money will be assigned across the three levels of the carding system; level one being for world class athletes, level two being for national athletes and level three being for emerging athletes. If athletes fail to perform, or NSO's lose their allocated funding support due to a lack of success (i.e. failing to produce athletes who achieve on the international sporting stage), athletes risk having any or all of their support revoked or reduced. Currently there are around four hundred and eighty athletes listed as part of the HPSNZ carding system (carded athlete list n.d.).

Although, many within the sporting community argue that elite development programmes, such as athlete carding, provide much needed support for emerging sporting talent, others question the cost to athletes' personal, emotional and social development. Specifically, whilst the carding system was developed with the intention of ensuring that New Zealand's athletes fulfil their potential both on and off the sports field, research suggests that the special attention and perceived reward that programmes like athlete carding provide, further entrenches, rather 
than reduces, reliance upon the athlete role (Henry 2013, Murphy et al. 1996, Ryan 2015). Additionally, as the criterion for sustained involvement within the system is contingent upon continued displays of athletic prowess, the programme risks reinforcing the sport ethic, and thus again encourages an exclusive focus on ones' athletic identity. Consequently, the way in which the carding programme is currently being delivered potentially counteracts one of its intended goals. Despite this, HPSNZ has ignored how its actions influence the lived experiences of New Zealand's carded athletes, beyond the impact upon their athletic potential. New Zealand's elite athletes therefore appear to be a previously silenced population, with little effort being made to discover what these individuals actually encounter as part of the carded athlete programme. The current project therefore provides a much needed opportunity for athletes to speak about a programme which has become central to many of their lives.

\section{Methods}

This research is framed within the interpretive paradigm where it is important that individuals represent their worlds, views and opinions from within their own repertoire of words and ideas (Kvale 1996). In particular, this paradigm views perceptions of self and identity as being socially constructed and recognises the influence that an individual's socio-cultural and historical context play in shaping their life experiences (Cresswell and Poth 2017, Smith and Sparkes 2009). As personal accounts are therefore considered to involve fluid and subjective multiple realities (Smith 2017, Sparkes and Smith 2014), the current project is not shaped by a single, overarching theoretical framework, rather it employs an interpretivist lens to engage with those perspectives and meanings portrayed by New Zealand's high performance athletes. 
This notion of personal meaning is central to the interpretivist approach, consequently, a series of one-to-one semi-structured interviews were considered to be the most effective way to capture athletes' respective experiences within the carding context. In an effort to produce information rich personal accounts, the researcher also used the process of purposeful sampling in order to ensure strong relationships existed between the research questions and the research participants (Patton 2008, Smith 2017). Accordingly, athletes who had been, or continued to be, carded for at least two years were deemed to be most suited to provide insight into life within this programme. This criterion was chosen because most NSOs assessed their carded athlete status and service provision on an annual basis. As such, athletes who had at least two years of experience within the carding system were guaranteed to have been through this procedure (at least once), and thus had sufficient lived experience and knowledge of the entire carding system under investigation.

\section{Participants}

In order to gain access to the carded athlete population the researcher approached a selection of ten national sporting organisations. These sports were chosen to ensure that the eventual research sample represented athletes from both team and individual sports, as well as what HPSNZ terms as 'priority' and 'non-priority' sports. Eight of the contacted sports agreed to allow their athletes to be accessed as part of the study. Carded athletes from these sports were then e-mailed and invited to participate in the research project. Those athletes who volunteered to take part were contacted by the researcher to arrange a convenient time and place to conduct their initial interview.

A total of 17 (ten female and seven male) carded athletes eventually took part in the investigation. These participants ranged in age from $17-45$ years $(M=27.7$ years $)$ and had, 
on average, 13.35 years of competitive experience in their chosen sport. Each of the participants had in the past represented, or continued to represent, New Zealand in their chosen sporting code, and had at least two years $(M=3.94$ years $)$ of experience within the carded athlete programme. The sample comprised of one athlete from lawn bowls, two athletes each from basketball, cricket, cycling, field hockey, rugby, and squash and four athletes from the sport of rowing.

\section{Procedure}

In order to ensure the athletes had adequate opportunity to share and reflect upon their experiences within HPSNZ's carded athlete programme participants were invited to take part in two semi-structured face-to-face interviews over a period of six months. Employing two points of data collection not only allowed the researcher time to build stronger relationships with the research participants, but also provided athletes with an opportunity to reflect upon their initial responses whilst continuing to live within the carding system. All but four of the 17 athletes took part in both rounds of interviews. Of the athletes who were unavailable to complete the second phase of the data collection, three were overseas competing or too busy during the timing of the second round of interviewing, whilst the remaining athlete purposefully chose not to take part in the follow-up interview. As these reasons primarily fell outside of the athletes' control the researcher, after discussions with the athletes concerned and in accordance with the informed consent process, decided that initial interview data collected from each of these individuals would still be included within the final research analysis.

The initial interviews lasted between one to two hours per athlete, whilst the follow-up interviews lasted between one and one and a half hours per athlete. Both of the interviews were guided by a series of open-ended and additional probative questions developed for the purpose 
of this investigation. These questions served as a guide only and were developed on the basis of a literature review (e.g., Brewer et al. 1993, Carless and Douglas 2013, Houle et al. 2010, Sparkes 1988) and the study's underlying research questions. In particular, these guides were created in order to gather an holistic perspective of life as a carded athlete and focused on athlete's perceptions of how being part of the carding system has impacted their identity development both in, and outside, of the sport environment The open-ended nature of the interview process invited each participant to tell their stories and ensured that a more comprehensive coverage of the topics under investigation could occur, whilst still maintaining the individualistic nature of each participant's account (Chenail 2011). As the interviews evolved clarification and elaboration probes were also used to refine participant meaning and further enrich athletes' accounts.

\section{Analysis and Interpretation}

Each of the athletes' interviews were recorded and then transcribed verbatim, with participants given pseudonyms to protect their confidentiality. Before beginning the data analysis procedure each of the interviews were read through several times by the researcher to ensure her familiarity with each participant's individual stories (Thomas and Hodges 2010). These transcripts were then systematically explored for meaning and context during an inductive thematic analysis (Braun and Clarke 2006). Following steps outlined by Lieblich et al. (1998) this process involved dissecting each participant's interview in order to identify descriptive themes from within the data itself. These themes were then reviewed and reorganised, as needed, to ensure that they remained both internally homogenous yet externally distinct (Deal and Camire 2016). In so doing the researcher was able to identify patterns in the way in which carded athletes talked about themselves and their experiences within the carding programme. 
Preliminary descriptive reports, outlining initial interpretations of the athletes' individual transcripts, were then provided to each participant. This procedure allowed each individual to review and reflect upon the accounts that they had provided, whilst simultaneously exploring the researcher's interpretations of these experiences (Sparkes and Smith 2014, Smith and McGannon 2017). Each athlete was then able to amend, or add to, any of their responses or these interpretations. Although the researcher does not attest that this process enhanced the credibility of the data analysis, it did provide a further opportunity to engage with research participants in a reflexive manner (Burke 2017, Smith and McGannon 2017). Upon receipt of each athlete's checked documents the researcher searched for further themes and meaning before collating the athletes' individual stories into a collective account for each significant theme. Throughout this process the researcher followed Guba and Lincoln's $(1981,1982)$ seminal work on trustworthiness and employed a variety of strategies to safeguard the quality of both the data collected and the conclusions reached. These strategies included keeping a reflexive journal and engaging a colleague to conduct an audit trail of the collected data and researcher's overall interpretations. Additionally, whilst not all participants contributed to each and every theme, the resulting topics represented those stories that were common to the majority of athletes. These themes will be explored in more detail during the following section.

\section{Results and Discussion}

The current research project provides insight into the lived experiences of a previously silenced, yet growing population of New Zealand athletes and presents carded athletes' perspectives on how this elite sport system has impacted their identity construction. Specifically, the research examines how expectations related to the normative sport ethic, and 
process of performance-based funding, encourage the prioritisation of a sport-only identity, and explores ways in which athletes challenge the pervasiveness of this identity.

\section{Entangled within the Athlete Role}

The prevalence of the athlete role was evident throughout each of the athletes' accounts of life within HPSNZ's carded athlete programme. Whilst many athletes willingly devoted their lives to pursuing sporting goals, at least some of this commitment was driven by perceived external pressures. Specifically, when roles like those occupied by the carded athletes exist, they do so based upon shared meaning and behaviours (Stryker 2008, vom Lehn and Gibson 2011). Consequently, upon entering the carding system athletes were confronted with the numerous expectations and entanglements which accompanied this specific role enactment. These expectations then needed to be navigated as part of the athletes' daily lives. For instance rugby player Holly explained, '[Because you are an elite athlete] people expect you to get up at the crack of dawn and... to train for four or five hours a day and be completely focused on your sport all the time'. Thus, when establishing the reputation of being an elite athlete, many of the interviewed athletes sought to perpetuate an image of self-sacrifice and overt dedication to their sporting selves.

Previous research (e.g., Douglas and Carless 2009, Hickey and Kelly 2008, Maguire 1991), has suggested that widespread acceptance of these normative behaviours leaves athletes so focused on establishing, and then maintaining the athlete role, they fail to question the ideas and values put forward by those in charge of these sport environments. This risk is particularly concerning given that many carded athletes looked to embrace, or at the very least passively accept, the athlete role rather than challenge its solitary existence. For instance, despite already 
being devoted to her sport for a number of years, Beth, a fresh faced novice within New Zealand Rowing's high performance squad revealed;

There are times when I sit out there and you are rowing along and I think to myself it is amazing that so much of my time is taken up doing this (laughs)... And I still think “Oh, I'm a rower!”... It's just surprising... We are definitely rowers... but there are times when you think "man, how did I get here, how did I turn out to be a rower?"

This lack of reflection was most apparent in those athletes, like Beth, who were new to the carding programme, or at the peak of their careers. Hughes and Coakley (1991) referred to such behaviour as positive deviance, or an excessive over-conformity to the norms and values of elite sport culture. Such behaviour is compounded by the fact that those in charge of sport environments control access to necessary resources, exposing athletes to a complex web of power relations, and expect them to embrace the role of compliant participant (Johns and Johns 2000). Whilst such displays benefit managers, sponsors and coaches whose focus is on winning and achievement, Maguire (2004) suggests this cultural practice fosters these power differentials and further perpetuates athletes' inability to exert control over their own sport experience.

Indeed, accounts from newly carded athletes suggest that a range of entities (i.e. HPSNZ, NSO's, coaches etc.) struggled for control over these individuals' lives, regulating their behaviour and normalising the sport-only identity position. Seemingly unaware of the inequitable power balance at play, most of the athletes fully trusted the advice of the authority figures within their sport and readily accepted that a singular sport focus was necessary in order to ensure on-going performance gains. Echoing the sentiments made by elite athletes in other 
studies conducted across the globe (e.g., Aquilina 2013, Butt and Molnar 2009, Kelly and Hickey 2010, Lavallee and Robinson 2007), they embraced the myth that effort, hard work and commitment would eventually get them everything they desired, thus actively restricting their personal development in areas outside of the elite sport environment. 21 year old cyclist Cam stated matter-of-factly, '[Sport] is my number one priority, I'm not really thinking about anything [else]... You are better off [that way]... You have less distractions'. Likewise, having freely admitted that she had very little influence over her daily schedule, Beth accepted that most major decisions in her life were now controlled by '[what] people [believe] we need to do to achieve our goals'.

Whilst positive deviance was most prevalent amongst the carding newcomers, it was apparent that all athletes in the study looked to those in charge of their sport environments to clarify the expectations required of carded athletes. With athletes exhibiting minimal resistance, coaches and NSO's strongly influenced whether they would, or could, choose to participate in more than just their athletic pursuits. In some incidences these support staff outwardly encouraged athletes to develop lives away from their sport environment. Nick shared '[Our] coach ... would say "See as much as you can, do as much as you can... Go out and have a good time"... He tries to get that balance [for us]'. More often than not, however, coaches and managers were seen as being unsupportive of athletes pursuing interests outside of elite sport. Recalling a conversation with his high performance manager Ben revealed, 'He said... "They don't give out Olympic medals to people with balanced lives"'. Additionally, newcomer Beth felt that the sheer logistical difficulty of taking part in activities outside of her sport showed New Zealand Rowing's failure to consider athletes' lives beyond what was necessary for their sport. She admitted that at any stage her coach 'could just say "oh, we are now going to start training three times a day"... [Which means] you can't [really] do anything except for rowing 
because... you have to be available... [So] you [end up living] session to session'. Such displays left athletes with little doubt that they should be prioritising their athletic goals over personal development in other life areas, thus promoting the social norms associated with the competitive sport environment (Carless and Douglas 2009, 2012, Hughes and Coakley, 1991).

\section{Implications of Carding and Performance-Based Funding}

The athletes' identities were also consolidated by expectations of carded athletes' time and energies. Upon reaching their carded status all athletes reported that their training requirements grew. For instance cyclist Jamie stated, 'Before I became carded I just trained... and I did okay... But now all this technical stuff is coming in... and I have got [fitness] testing and all sorts of things that I never had before, so it is more stressful'. This scientific approach to athletic training reflects the overarching performance-efficiency model that underpins the normative sport ethic (Collins and Cruickshank 2012, Maguire 2004). Tannsjo (2000) suggested that by embracing this paradigm the carding system risks reducing individuals' performances to qualities that can be manipulated through modern science and medicine. In particular, such efforts dehumanise the sport process and lead athletes to prioritise immediate rather than long-term goals and their physical rather than psychological needs. Demonstrating this shifting focus Mia shared, [You begin to think] if other people are taking it seriously enough to provide you with these services then ... you need to treat it more professionally'. Like many of the athletes, Mia recognised the weight of expectation that came with her carding status.

Indeed, as all future funding and selection decisions were explicitly linked to reaching HPSNZ's performance expectations, most athletes felt that they were sent a clear and explicit 
message about reaching and maintaining peak performance. Mia described this pressure when she recounted her experiences at a recent Commonwealth Games. Knowing her basketball team needed to achieve a medal in order to secure future funding she stated, 'I think our semi-final was almost more important than the final... because we had to medal. I feel like we ran to death... seven players in that semi-final because we were going to leave absolutely nothing to chance'. Likewise, twenty nine year old rugby player Lucy associated enhanced scrutiny and performance monitoring with the funding supplied to athletes as part of the carding programme. She was mindful that her status within the carding system was based upon her proven and/or perceived athletic potential and although she greatly appreciated the support that her carded status afforded her, she shared, 'The support is not unconditional, that's for sure. You know there is pressure there'.

With NSO's revenue contingent on obtaining international sporting success (e.g. a top three finish during pinnacle events such as the Olympic Games), some researchers (e.g., Henry 2013, Murphy et al. 1996) have suggested that coaches and administrators are far more determined to achieve positive results than focus on athlete welfare. Indeed, as the livelihoods of these individuals are dependent upon success on the international sporting stage, athletes' long term needs typically come second to the more immediate goal of winning gold medals. Therefore, despite HPSNZ championing the ideals of holistic athlete development, its policy of performance-based funding severely limits the likelihood of this outcome actually occurring. This failure to support life outside of the sport environment thus leaves athletes further entrenched within the athletic lifestyle (Brewer et al. 1993, Lavallee and Robinson, 2007, Price et al. 2010, Wilding et al. 2012). For the most part, however, athletes considered performance pressure to be an accepted part of the carding process and likened it to workplace scrutiny found elsewhere within society. As such they appeared to readily accept the conditions 
associated with the support on offer. For instance, seventeen-year-old age group representative, Kate, felt that Basketball New Zealand's expectations on her time and effort was appropriate because 'They are putting money into you so you had better work well for them'. Similarly Jan, an older athlete, acknowledged that '[Carding] is not just free money that is given out just because you play sport at certain level, you have to have a performance measure against it'.

Additionally, despite some dismay over the pressures associated with becoming carded, no athlete was willing to part with their services in order to reduce these expectations. Instead, all of the athletes expressed a desire to enhance, or at least maintain, their current levels of support. Such acts of deference therefore reveal a desire to secure favour with NSO's, who wield disciplinary power within NZ's elite sport environment. Although such actions could be construed as an apparent acceptance of the current programme, it is more likely a reflection of the lack of agency and on-going surveillance that permeates athletes' lives once part of the carding system. With their athletic progress constantly under a microscope, Casey et al. (2016) suggests that athletes' behaviour becomes increasingly self-regulated as they embrace the rules and norms of the dominant sport ethic. In so doing athletes are adopting one of the few mechanisms they see available in order to retain their place within the limited space of the carding system.

\section{Challenging the Sport Ethic}

It was not until athletes entered the later stages of their careers that they began to question why the carding programme encourages such a narrowed athlete identity. Whilst they agreed that achievement in top level sport undoubtedly required commitment and a certain amount of sacrifice, some of the more seasoned carded athletes questioned whether a singular focus upon sport was indeed beneficial to obtaining elite level success. In fact, many of these individuals 
described greater levels of enjoyment and better performances after adjusting the place and value that sport held within their lives. Peter shared,

You need to have some little job or something that keeps you away from it... [I started to achieve and enjoy my sport more when I was] having a life outside of hockey. Finding time to work and see my wife... Not having hockey control my life.

Such results provide further support for Carless and Douglas's (2013) assertion that athletic success is still possible when physical training is pursued in conjunction with personal development in other life areas. Similarly, the athletes' experiences strengthen the position of researchers (e.g., Alfermann and Stambulova 2007, Carless and Douglas 2012, 2013, Lally 2007, Miller and Kerr 2003, Stambulova 2012) who suggest that athletic performances benefit from a more holistic approach to athlete development.

Indeed, seasoned carded athletes suggested that sacrificing a multi-dimensional identity in the name of sporting success was neither advantageous, nor sustainable. Faced with the reality that their carding support was not always assured, and having witnessed the futile nature of this system of expendability, many of these individuals had instead begun planning for their eventual sport retirement. Challenging the underlying sport ethic, these athletes actively pursued interests and experiences away their sporting endeavours. Some chased vocational opportunities in addition to their athletic dreams, whilst others sought out friends, other sports and/or higher education in order to bring some balance to their lives. Lucy, a rugby player working towards her Master's degree felt that, 'It is good to just get away from sport sometimes... for my mind to be able to just switch off and talk about something else that is 
completely random or irrespective of what it is that I do'. Whilst hockey player Helen acknowledged,

[After years of being constantly on the fringe of New Zealand selection I eventually] tried to have something else that I was trying to achieve so that when I came out the other end I had a back stop, or if I got dropped or I got injured... [Basically I thought it was important that] if your sporting career ends all of a sudden, then you have got something else that you do.

By seeking out alternative roles and experiences in conjunction with their sport participation, these carded athletes were able to connect with a life outside of the sport, whilst still maintaining their athletic identity. This process enabled athletes to slowly reduce their reliance on the sporting aspect of their identity and instead develop a more well-rounded sense of self. In so doing, the athletes became far better equipped to transition into a life without elite sport (Lavallee and Robinson, 2007, Samuel and Tenenbaum 2013, Stephan et al., 2003). In order to assist in this process Holly challenged those in charge of sport environments to better integrate these alternative aspects of athletes' lives. She stated, '[They need to] understand that you have got other parts of your life and... that we can not sacrifice everything for our sport'.

Stambulova et al. (2009) suggested that this process is particularly important as retirement from sport is one of the inevitable transitions athletes will face during the course of their careers. However, as previously stated, most newly carded athletes gave little attention to what their lives might be, or become, beyond their sporting participation. Whilst previous research (e.g., Carless and Douglas 2013, Stambulova et al. 2009, Wylleman and Lavallee 2004) has noted that athletes' typically channel their energies towards those transitions and requirements 
immediately relevant in that given moment, this reluctance to look to the future remains rather concerning. It is therefore crucial that those in charge of elite sport environments address this issue in order to ensure all athletes are prepared for the inevitable end of their sporting participation. Additionally, differences in athlete readiness suggests that education and assistance in the area of personal development should be tailored to the athlete's specific needs, be regularly evaluated and more importantly be based upon the athlete's career life cycle.

\section{Conclusion}

Since its establishment in 2002, HPSNZ's carded athlete programme has played a significant role in the development of New Zealand's elite athletes. Although introduced with the intention of assisting athletes to develop both on, and off, the field little is known about the impact that this sport development model has actually had on the athletes involved. The current research project therefore sought to rectify this gap in literature. A series of interviews with selected carded athletes revealed that whilst the carding programme has provided increased opportunity to develop athletes' sporting talents, it has also brought about increased scrutiny and performance expectation. Such pressures have shaped the role and place that elite sport holds within carded athletes' lives. In particular, the carding programme has ensured that athletes consolidate a firm athlete identity, often at the expense of achieving a well-balanced and holistic sense of self. This outcome contradicts the underlying philosophy upon which the carding programme was built, and can leave athletes facing formidable transitions into postsport careers upon the cessation of their elite sport involvement.

If holistic development is to be given greater credence within the carding programme, critical attention must be directed at the culture of New Zealand's elite sport system. In particular, 
HPSNZ needs to re-examine the way in which it supports and funds elite sport programmes. Specifically, more emphasis should be placed upon enhancing personal development alongside sporting performance when allocating funds to national sporting organisations. Additionally, HPSNZ needs to engage in a wide-reaching education programme that promotes the importance of developing a well-balanced sense of self alongside one's sporting potential. Given that there appeared to be some resistance by newly carded athletes to prepare for life beyond their sporting careers, and the fact that sport retirement cannot always be planned for (e.g. in the case of injury or de-selection), any education programme should also include strategies which promote personal development throughout all stages of an athlete's sporting career.

Athlete accounts also indicate that whilst the current sport development model offers athletes some level of responsibility (i.e. performance accountability), it provides very little in the way of self-autonomy. In particular, the carding model appears to hand control of an athlete's development over to respective sport administrators and/or coaches. These individuals are then tasked with setting out performance plans, often with little regard for how these actions may affect the lives of athletes under their control. Therefore in order to move personal development to the forefront of this agenda athletes need to be considered as 'whole' people, rather than just for their sporting talents. Additionally, efforts need to be made to regain some of the selfdetermination that appears to be lost within the current model.

The willingness for athletes to unquestioningly accept their current fate does, however, demonstrate the prevailing sport ethic and its underlying belief that such behaviours are 'just part and parcel of living the elite sport lifestyle'. It remains unclear whether this absence of personal reflection is an orchestrated strategy of denial in order to cope with the limited 
freedoms athletes experience within their sporting lives, or an ignorance stemming from the belief that coaches and NSOs are working with athletes' best interests at heart. What we do know, however, is that as more carded athletes are seen to relinquish control over their sporting selves, they further perpetuate the belief that such behaviour is the appropriate norm for our elite sportspeople. As such, those in charge of these environments are gaining compliant, and seemingly complacent, athletes who further entrench those role-related behaviours that do not always favour the individuals whom they portray. This performance culture therefore needs to be challenged by athletes, coaches and sport administrators alike. Such attempts will first require those in charge of sport environments to reflect upon how their own behaviours reinforce this lack of power, before then encouraging athletes to embrace identities that extend beyond this prevalent narrative.

Finally, there is scope for further investigations to incorporate a more critical lens when analysing the experiences of New Zealand's carded athletes. In particular, it would be valuable for researchers to examine how the underlying principles of power and surveillance have shaped carded athletes' identities in greater depth. Specifically, Ronkainen et al. (2016) suggests that in order to facilitate and expand upon viable alternatives we must first deconstruct those discourses which currently constrain athletes' identities. In so doing, researchers could also draw upon the views and experiences of other entities within the carding programme (e.g., coaches, high performance managers etc.). Whilst such viewpoints were not the focus of the current research project, these insights would further explore the perceived lack of agency that was evident within the stories collected during this investigation. Such research has the potential to strengthen the body of research in this area and facilitate the emergence of 
alternative career development programmes that prioritise the well-being of athletes alongside their on-field performances.

\section{Acknowledgements}

I would like to thank the participants who generously volunteered their time to make this research project possible. I also thank Sport and Recreation New Zealand who helped to fund this research.

\section{References}

Albion, M. J., and Fogarty, G. J., 2005. Career decision making for young elite athletes: Are we ahead on points? Australian journal of career development, 14, 51-62. doi:10.1177/103841620501400108

Alfermann, D., and Stambulova, N., 2007. Career transitions and career terminations. In G. Tenebaum \& R. Eklund (Eds.), Handbook of sport psychology (pp. 712-733). New York, NY: Wiley.

Aquilina, D., 2013. A study of the relationship between elite athletes' educational development and sporting performance. The international journal of the history of sport, 30(4), 374392. doi:10.1080/09523367.2013.765723

Archer, D. E., 2010. Preparing for exit from sport: A phenomenological examination of the pretransition experiences of division I female intercollegiate athletes. (Unpublished doctoral thesis). Oklahoma State University, USA. Braun, V., and Clarke, V., 2006. Using thematic analysis in psychology. Qualitative research in psychology, 3, 77-101. doi:10.1191/1478088706qp063oa 
Brewer, B. W., Van Raalte, J. L., and Linder, D. E., 1993. Athletic identity: Hercules' muscle or archilles heel? International journal of sport psychology, 6, 279-288.

Burke, S., 2017. Rethinking 'validity' and 'trustworthiness' in qualitative inquiry. In B. Smith \& A. C. Sparkes (Eds.), Routledge handbook of qualitative research in sport and exercise (pp.330-339). London: Routledge.

Butt, J., and Molnar, G., 2009. Involuntary career termination in sport: A case study of the process of structurally induced failure. Sport in society: Cultures, commerce, media, politics, 12, 240-257. doi:10.1080/17430430802591027

Carded athlete list, n.d. Retrieved from http://hpsnz.org.nz/athletes/carding Accessed 02.10.16.

Carless, D., and Douglas, K., 2009. "We haven't got a seat on the bus for you" or "all the seats are mine": Narratives and career transition in professional golf. Qualitative research in sport and exercise, 1(1), 51-66.

Carless, D., and Douglas, K., 2012. Stories of success: Cultural narrative and personal stories of elite and professional athletes. Reflective practice: International and multidisciplinary perspectives, 13(3), 387-398. doi:10.1080/14623943.2012.657793

Carless, D., and Douglas, K., 2013. "In the boat" but "selling myself short": Stories, narratives, and identity development in elite sport. The sport psychologist, 27, 27-39.

Casey, M., Mooney, A., Smyth, J., and Payne, W., 2016. 'Power, regulation and physically active identities': The experiences of rural and regional living adolescent girls. Gender and education, 28, 108-127. doi:10.1080/09540253.2015.1093098

Chenail, R. J., 2011. Interviewing the investigator: Strategies for addressing instrumentation and researcher bias concerns in qualitative research. The qualitative report, 16, 255-262. Coakley, J., and Donnelly, P., 1999. Inside sports. London: Routledge. 
Collins, D., and Cruickshank, A., 2012. 'Multi-directional management': Exploring the challenges of performance in the World Class Programme environment. Reflective practice: International and multidisciplinary perspectives, 13(3), 455-469.

Cosh, S., et al., 2013. Career transitions and identity: A discursive psychological approach to exploring athlete identity in retirement and the transition back into elite sport. Qualitative research in sport, exercise and health, 5(1), 21-42. doi:10.1080/2159676X.2012.712987

Cosh, S., and Tully, P. J., 2014. All I have to do is pass“: A discursive analysis of student athletes ' talk about prioritising sport to the detriment of education to overcome stressors encountered in combining elite sport and tertiary education. Psychology of sport and exercise, 15, 180-189. doi:10.1016/j.psychsport.2013.10.015

Creswell, J. W., and Poth, C. N., 2017. Qualitative inquiry and research design: Choosing among five approaches. Thousand Oaks, CA: Sage publications.

Deal, C. J., and Camire, M., 2016. University student-athletes' experiences of facilitators and barriers to contribution: A narrative account. The qualitative report, 21(11), 2087-2102.

Donnelly, P., and Young, K., 1988. The construction and confirmation of identity in sport subcultures. Sociology of sport journal, 5, 223-240.

Douglas, K., and Carless, D., 2009. Abandoning the performance narrative: Two women's stories of transition from professional sport. Journal of applied sport psychology, 21, 213-230. doi: 10.1080/10413200902795109

Guba, E., and Lincoln, Y., 1981. Effective evaluation. San Francisco, CA: Jossey-Bass.

Guba, E. G., and Lincoln, Y. S., 1982. Epistemological and methodological bases of naturalistic inquiry. Educational communications and technology journal, 30(4), 233252. 
Henry, I., 2013. Athlete development, athlete rights and athlete welfare: A European Union perspective. The international journal of the history of sport, 30(4), 356-373. doi: $0.1080 / 09523367.2013 .765721$

Hickey, C., and Kelly, P., 2008. Preparing to not be a footballer: Higher education and professional sport. Sport, education and society, 13(4), 477-494.

Houle, J. L. W., Brewer, B. W., and Kluck, A. S., 2010. Developmental trends in athletic identity: A two part retrospective study. Journal of sport behavior, 33, 146-159.

Hughes, R., and Coakley, J., 1991. Positive deviance amongst athletes: The implications of overconformity to the sport ethic. Sociology of sport journal, 8, 307-325.

Johns, D. P., and Johns, J. S., 2000. Surveillance, subjectivism and technologies of power. International journal for the sociology of sport, 35, 219-234.

Kelly, P., and Hickey, C., 2010. Professional identity in the global sports entertainment industry: regulating the body, mind and soul of Australian Football League footballers. Journal of sociology, 46(1), 27-44. doi:10.1177/1440783309337671

Kvale, S., (1996). Interviews: An introduction to qualitative research interviewing. London: Sage.

Lally, P., 2007. Identity and athletic retirement: A prospective study. Psychology of sport and exercise, 8, 85-99. doi:10.1016/j.psychsport.2006.03.003

Lally, P., and Kerr, G. A., 2005. The career planning, athletic identity, and student role identity of intercollegiate student athletes. Research quarterly for exercise and sport, 76(3), 275-285.

Lavallee, D., and Robinson, H., 2007. In pursuit of an identity: A qualitative exploration of retirement from women's artistic gymnastics. Psychology of sport and exercise, 8, 119141. doi:10.1016/j.psychsport.2006.05.003 
Lieblich, A., Tuval-Mashiach, R., and Zilber, T., 1998. Narrative research: Reading, analysis and interpretation. London: Sage.

Litchke, L. G., et al., 2012. Personal meaning of wheelchair rugby participation by five male athletes. Therapeutic recreation journal, 46, 26-41.

Louder, J., 2003. Maintain your identity. Bicycling Australia, 14(2), 86-87.

Maguire, J., 1991. Human sciences, sport sciences, and the need to study people in "in the round”. Quest, 43(2), 190-206. doi: 10.1080/00336297.1991.10484021

Maguire, J., 2004. Challenging the sports-industrial complex: Human sciences, advocacy and service. European physical education review, 10(3), 299-322.

Miller, K. E., 2009. Sport-related identities and the "Toxic Jock". Journal of sport behavior, 32(1), 69-91.

Miller, P. S., et al., 2007. Athletic involvement and adolescent delinquency. Journal of youth and adolescence, 36, 711-723.

Miller, P. S., and Kerr, G. A., 2003. The role experimentation of intercollegiate student athletes. The sport psychologist, 17, 196-219.

Murphy, G. M., Petitpas, A. J., and Brewer, B. W., 1996. Identity foreclosure, athletic identity, and career maturity in intercollegiate athletes. The sport psychologist, 10, 239-246.

Patton, M. Q., 2008. Utilization-focused evaluation. Saint Paul, MN: Sage publications.

Price, N., Morrison, N., and Arnold, S., 2010. Life out of the limelight: Understanding the nonsporting pursuits of elite athletes. The international journal of sport and society, 1(3), 69-79.

Pummell, E., Harwood, C., and Lavallee, D., 2008. Jumping to the next level: A qualitative examination of within-career transition of adolescent event riders. Psychology of sport and exercise, 9(4), 427-447. doi:10.1016/j.psychsport.2007.07.004 
Ronkainen, N. J., Kavoura, A., and Ryba, T. V., 2016. Narrative and discursive perspectives on athletic identity: Past, present, and future. Psychology of sport and exercise, 27, 128137. doi: 10.1016/ j.psychsport.2016.08.010

Ryan, C., 2015. Factors impacting carded athlete's readiness for dual careers. Psychology of sport and exercise, 21, 91-97. doi: 10.1016/j.psychsport.2015.04.008

Samuel, R. D. and Tenenbaum, G., 2013. Athletes' decision-making in career change-events. The sport psychologist, 27, 78-82.

Smith, J. A., 2017. Interpretative phenomenological analysis in sport and exercise. In B. Smith \& A. C. Sparkes (Eds.), Routledge handbook of qualitative research in sport and exercise (pp.219-229). London: Routledge.

Smith, B., and McGannon, K. R., 2017. Developing rigor in qualitative research: Problems and opportunites within sport and exercise. International Review of Sport and Exercise Psychology. doi:10.1080/1750984X.2017.1317357

Smith, B., and Sparkes, A. C., 2009. Narrative inquiry in sport and exercise: What can it mean, and why might we do it. Psychology of sport and exercise, 10, 1-11. doi: 10.1016/j.psychsport.2008.01.004

Sparkes, A., 1998. Athletic identity: An Achilles' heel to the survival of self. Qualitative health research, 8(5), 644-664. doi:10.1177/104973239800800506

Sparkes, A. C., and Smith, B., 2014. Qualitative research methods in sport, exercise and health: From process to product. New York: Routledge.

Stambulova, N., 2012. Working with athletes in career transitions. In S. Hanton and S. Mellalieu, eds. Professional practice in sport psychology: A review. London, UK: Routledge, 165-194. 
Stambulova, N., et al., 2009. ISSP position stand: Career development and transitions of athletes. International journal of sport and exercise psychology, 7(4), 395-412. doi:10.1080/1612197X.2009.9671916

Stephan, Y., and Brewer, B. W., 2007. Perceived determinants of identification with athlete role among elite competitors. Journal of applied sport psychology, 19, 67-79.

Stephan, Y., et al., 2003. Repercussions of transition out of elite sport on subjective well-being: A one-year study. Journal of applied sport psychology, 15, 354-371.

Stryker, S., 2008. From Mead to a structural symbolic interactionism and beyond. Annual review of sociology, 34, 15-31.

Sturm, J. E., Feltz, D. L., and Gilson, T. A., 2011. A comparison of athlete and student identity for Division I and III athletes. Journal of sport behavior, 34, 295-306.

Tannsjo, T., 2000. Is it fascistoid to admire sports heros. In T. Tannsjo, \& C. Tamburrini (Eds.), Values in sport: Elitism, nationalism, gender equality and the scientific manufacture of winners (pp. 9-23). London, UK: E \& FN Spon Publishers.

Thomas, D. R., and Hodges, I. D., 2010. Designing and managing your research project: Core knowledge for social and health researchers. Thousand Oaks, CA: Sage Publications.

vom Lehn, D., and Gibson, W., 2011. Interaction and symbolic interactionism. Symbolic interaction, 34, 315-318. doi:10.1525/si.2011.34.3.315

Wainwright, S. P., Williams, C., and Turner, B. S., 2005. Fractured identities: Injury and the balletic body. Health, 9, 49-66.

Webb, W. M., et al., 1998. Athlete identity and reactions to retirement from sports. Journal of sport behavior, 21, 338-352.

Weiss, O., 2001. Identity reinforcement in sport: Revisiting the symbolic interactionist legacy. International review for the sociology of sport, 36(4), 393-405. doi:10.1177/101269001036004002 
Wilding, A. J., Hunter-Thomas, L., and Thomas, R., 2012. Sacrifice: The lonely Olympic road. Reflective practice: International and multidisciplinary perspectives, 13(3), 439-453. doi: $10.1080 / 14623943.2012 .670108$

Wylleman, P., and Lavallee, D., 2004. A developmental perspective on transitions faced by athletes. In M. R. Weiss (Ed.), Developmental sport and exercise psychology: A lifespan perspective (pp. 503-524). Morgantown, WV: Fitness Information Technology. 